\title{
An Implementation of Error Minimization Position Estimate in Wireless Inertial Measurement Unit using Modification ZUPT
}

\author{
Adytia Darmawan, Sanggar Dewanto, Dadet Pramadihanto \\ Politeknik Elektronika Negeri Surabaya \\ Jl. Raya ITS Sukolilo Surabaya 60111, Indonesia \\ Telp: 62315947280 Fax : 62315946114 \\ E-mail: adyt@pasca.student.pens.ac.id, \{sanggar,dadet\}@pens.ac.id
}

\begin{abstract}
Position estimation using WIMU (Wireless Inertial Measurement Unit) is one of emerging technology in the field of indoor positioning systems. WIMU can detect movement and does not depend on GPS signals. The position is then estimated using a modified ZUPT (Zero Velocity Update) method that was using Filter Magnitude Acceleration (FMA), Variance Magnitude Acceleration (VMA) and Angular Rate (AR) estimation. Performance of this method was justified on a six-legged robot navigation system. Experimental result shows that the combination of VMA-AR gives the best position estimation.
\end{abstract}

Keywords: Position Estimation, Modified ZUPT, Navigation System

\section{INTRODUCTION}

Inertial Measurement Unit (IMU) is an electronic component for measuring acceleration, velocity, or the attitude of the mechanical structure, using a combination of accelerometer sensor, gyroscope, and magnetometer. Generally, a sensor IMU has six degrees of freedom comprising 3-axis accelerometer and 3-axis gyroscope. IMU is a major component of the Inertial Navigation System (INS) on a land vehicle, water vehicle, aircraft, spacecraft, and missiles. The more expensive the price of the IMU will be more accurate specification of each sensor available on the IMU. IMU in Inertial Navigation System is used as the data source for position error compensation GPS data in the outdoor area. In the indoor area IMU position and orientation of the data will be processed further to get a better accuracy than other systems such as indoor localization technology electromagnetic, acoustic and visual [1].

IMU is generally equipped with a feature AHRS (Attitude and Heading Reference System). An AHRS is a mechanism to get the data the attitude and direction that occur by using sensors in the IMU fusion. Some AHRS algorithm triggered by Robert Mahony with Complementary Filter [2], Madgwick with Gradient Descent Algorithm[3], Premerlani \& Bizard's using 
Direction Cosinus Matrix [4]. Algorithms has advantages and disadvantages in terms of computing speed and accuracy of the resulting. Prices will affect the level of IMU sensors.

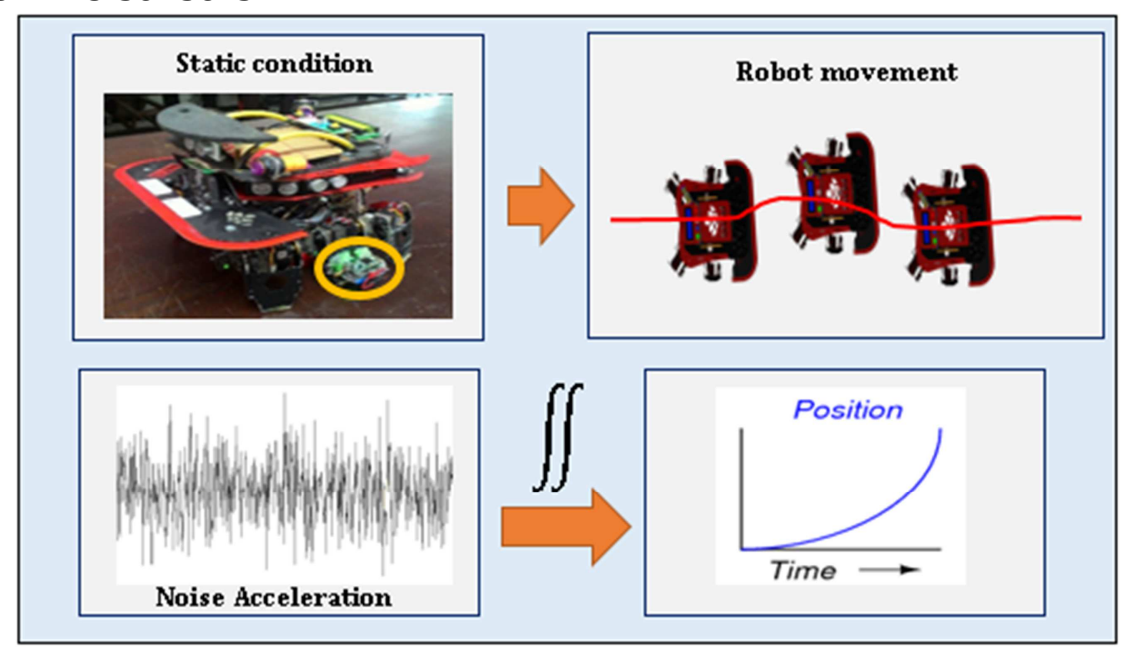

Figure 1. The smallest measurement errors result in an exponentially increasing error in the position estimation.

Small noise on the sensor Inertial Measurement Unit (IMU) will cause the position estimate error increases with time. Fortunately, a foot-mounted INS aided by the zero velocity update (ZUPT) technique can bound the navigation errors[5]. In the experiment, the movement detection method Zero velocity update (ZUPT) be modified by combining Magnitude Acceleration (MA) and Angular Rates (AR) estimate. Testing will be conducted on a foot-legged robot. A six-legged robot is different environmental condition with humans. Detection movement technique will be exploited here to get a good performance.

\section{RELATED WORKS}

In the university of Freiburg Germany, Fabian Hoflinger et al [1] was to reduce the size, weight and power consumption Micro-IMU. Micro-IMU was mounted on a shoe for detecting human movement. In the experiment Zhelong Wang et al [5], research a focus in stance-phase detection for reducing error position estimate for pedestrian navigation system. ZUPT used to reduce error in velocity estimation

Next, Slawomir et al [6] using kalman filter to achieve proper estimates of attitude and position signals that could be further used in unmanned aerial vehicle autopilots. In the experiment result shows that kalman filter deals with GPS accuracy decreases and magnetometer measurement noise. Rui Zhang et al [7] present's indoor localization and monitoring system for an emergency responder with modified Kalman filters for reducing magnetic field disturbance in indoor area. In the experiment result shows that was success to track people indoor in walking and running. 
Then, Kevin et al [8] has completed his research about design and use low cost inertial navigation system using an inertial measurement unit, digital compass, GPS and embedded computer system. In the result shows that power of GPS to aid in navigation is great. Low cost inertial navigation system made to have an accuracy of 10 meters and has an update rate of 1 Hz. Wang Jeen-Shing [9] using an IMU based pen (IMUPEN) for trajectory reconstruction and handwritten digit recognition applications. In the handwriting digit recognition application, the overall recognition accuracy is 90.4\% with a limited writing ambit and 94.6\% without any writing restriction. Oscar Laureano Casanova [10] has implement IMU and GPS sensors for tracking a navigation mobile robot. The error of positioning robots has as maximum 19,7 inch.

\section{ORIGINALITY}

Position estimation using an IMU is extremely difficult to achieve. This is because an accelerometer sensor in IMU is only able to provide a direct measurement of acceleration and the position must be derived from this through 'double integration'. The smallest measurement errors result in an exponentially increasing error in the position estimation. In this paper, we proposed to reduce error position estimating in wireless Inertial Measurement Unit (IMU) using modified Zero velocity Update (ZUPT). The position is then estimated using a modified ZUPT (Zero Velocity Update) method that was using Filter Magnitude Acceleration (FMA), Variance Magnitude Acceleration (VMA) and Angular Rate (AR) estimate. Performance of this method was justified on a six-legged robot navigation system.

\section{SYSTEM DESIGN}

Overviews of the system are shown in Figure. 2. Determination of the position estimation using an Inertial Measurement Unit (IAU) consists of two processes, among others orientation and position estimation Vibrations that occur on the robot cannot be predicted well in a state of static or in motion. Therefore, an error in the estimated position can only be reduced. The Direction Cosinus Matrix (DCM) output is a filtered rotation matrix. Results of the rotation matrix conversion did as quaternion. Conversion of a rotation matrix to quaternion is needed, to avoid the phenomenon of gimbal lock on euler angle. The next position estimation process is the separation between the linear acceleration by the acceleration of gravity affected. The result of the integration of linear acceleration obtained velocity. From the results obtained velocity is needed for the detection of the measured object at rest or in motion. This is necessary because the speed value is updated when static condition. Results ZUPT then does the integration with respect to time to obtain a position estimate. 


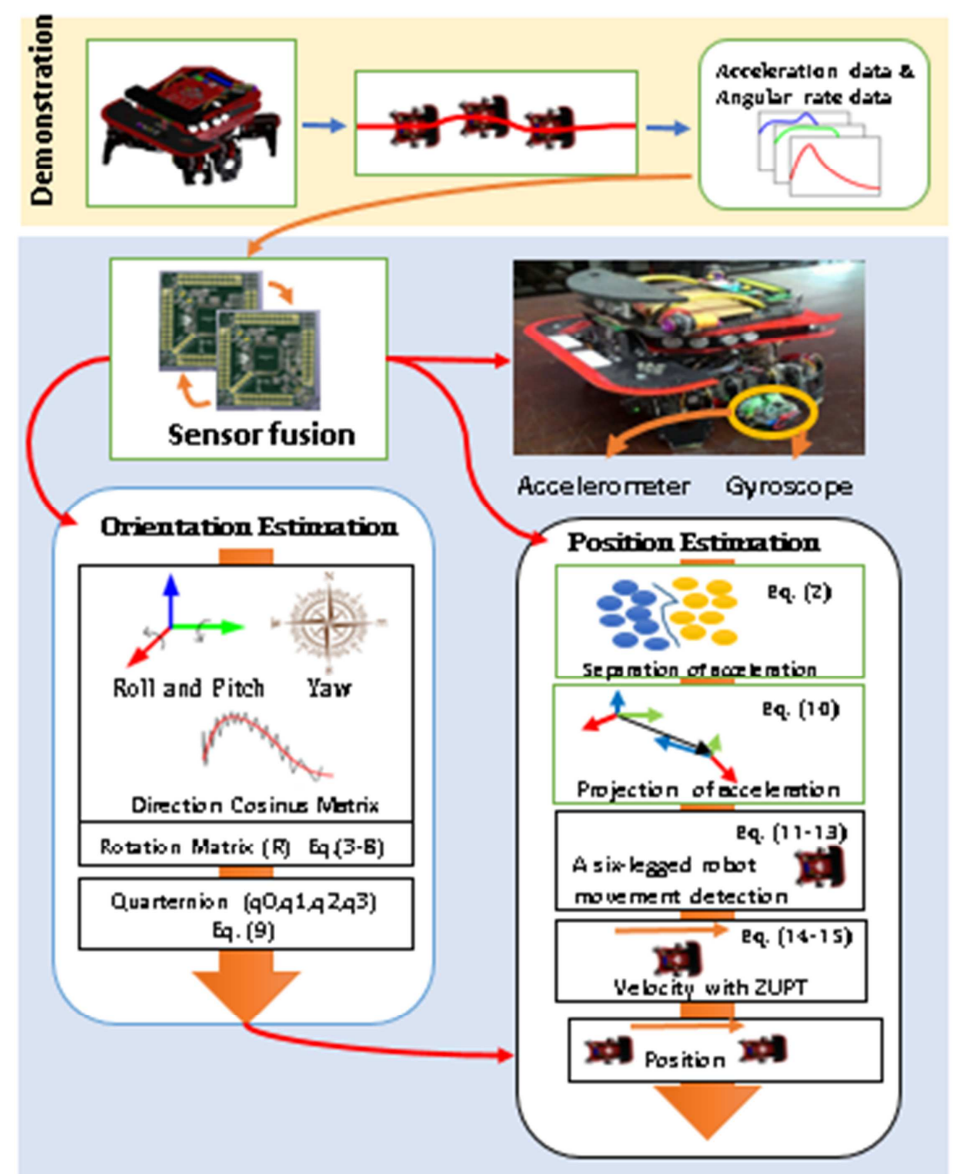

Figure 2. Overview system position estimation on a six-legged robot in navigation system using Wireless IMU

\subsection{Prototype and Hardware Setup}

IMU in experiment has a type processor MK20DX256VLH7 with overclockable $96 \mathrm{Mhz}$. Data acceleration and angular rate sensors using radio frequency transmitted at a frequency of 433Mhz. IC L3G20 is a type sensor accelerometer. IC LSM303 is an integrated IC gyroscope with a magnetometer.

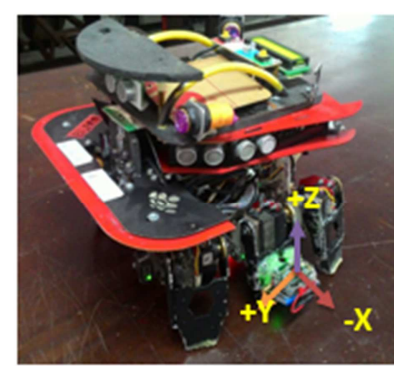

a)

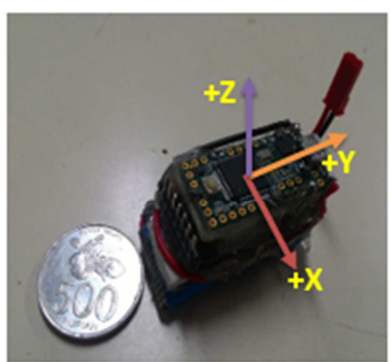

b)

Figure 3. Prototype Wireless IMU and hardware setup : (a) Hardware Setup W-IMU in Legged Robot, (b) Prototype Wireless Inertial Measurement Unit. 
Prototype IMU on the legged robot is placed on robotic legs. Robot move straight along the wall. Distance start to finish about 3 meters, the distance the robot as it moves toward the wall no more than $45 \mathrm{~cm}$ as shown in Figure. 4.

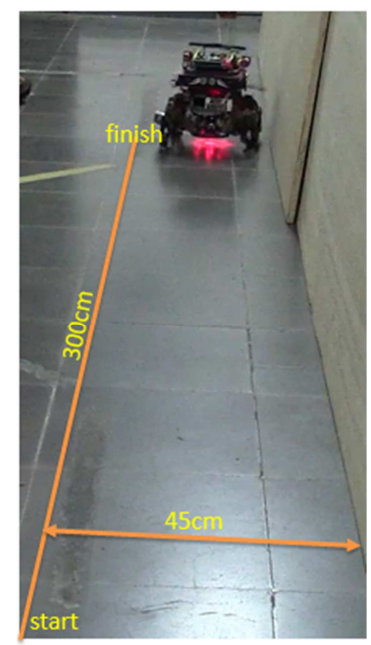

Figure. 4. Experiment Setup

\subsection{Linier Acceleration Estimation}

Linear acceleration is needed as a source of data to estimate the position. By using low cost IMU will require considerable processing stages in estimating the position. Accelerometer data $\left(\ddot{x}_{x, y, z}\right)$ consists of three types of data, such as linier acceleration $\left(\ddot{x}_{l x, l y, l z}\right)$, gravity acceleration $\left(\ddot{x}_{g x, g y, g z}\right)$ and noise $(u)$. Accelerometer data can represent in this equation:

$$
\ddot{x}_{x, y, z}=\ddot{x}_{l x, l y, l z}+\ddot{x}_{g x, g y, g z}+u
$$

The noises $(u)$ have several types, among others random noise, bias and white noise. Noise can be obtained from the datasheet or by observation to obtain the characteristics of the sensor. Equation 1 can be concluded that the linear acceleration can be obtained as in Equation 2.

$$
\ddot{x}_{l x, l y, l z}=\ddot{x}_{x, y, z}-\ddot{x}_{g x, g y, g z}-u
$$

Acceleration linier accuracy depends on the accuracy of estimation of the gravitational acceleration and noise.

\subsection{Gravity Acceleration Estimation}

Accuracy of linier acceleration estimates is highly dependent on the accuracy gravity estimation algorithm. Prior to estimate the acceleration of gravity orientation processing performed to determine the attitude of the object in traking. In experiment, we use premerlany and bizard to get the data orientation and modified to represent quarternion to eliminate the 
phenomenon of gimbal lock on Euler angle. Overviews of the DCM methods are shown in Figure 5.

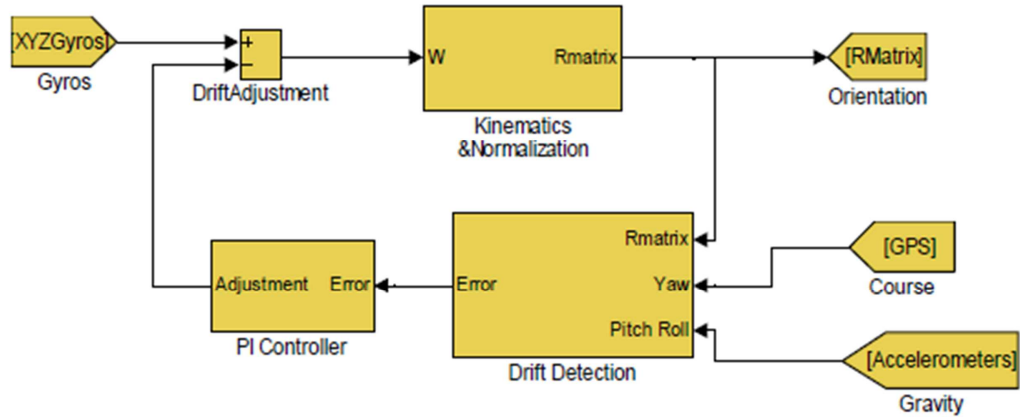

Figure 5. Overviews of the Direction Cosinus Matrix(DCM) methods [4]

Gyro sensor as the orientation of data resources. The results of the kinematic and normalization obtained rotation matrix (R) for conversion into Euler Angle. Due GPS in indoor areas not covered so that the data heading or yaw obtained from the magnetometer sensor data processing. Accelerometer combination treatment with the magnetometer used as a data source for correction of drift that occurs in gyroscope sensors. Error generated will be in though as the data gyro drift adjustment for subsequent processing. The following algorithms from[4]: Accelerometer data can represent in this equation:

$$
\begin{aligned}
& R^{T}=\left(r_{x} r_{y} r_{z}\right) \\
& \lambda_{r}=\frac{1}{2} r_{x} r_{y} \\
& r_{x}^{\prime}=r_{x}-\lambda_{r} r_{y} \\
& r_{y}^{\prime}=r_{y}-\lambda_{r} r_{x} \\
& r_{x}^{\prime \prime}=\frac{1}{2}\left(3-\left|r_{x}^{\prime}\right|^{2}\right) r_{x}^{\prime} \\
& r_{y}^{\prime \prime}=\frac{1}{2}\left(3-\left|r_{y}^{\prime}\right|^{2}\right) r_{y}^{\prime} \\
& r_{z}^{\prime \prime}=r_{x}^{\prime \prime} r_{y}^{\prime \prime} \\
& R^{T^{\prime}}=\left(r_{x}^{\prime \prime} r_{y}^{\prime \prime} r_{z}^{\prime \prime}\right)
\end{aligned}
$$

1. Calculate a correction magnitude $\left(\lambda_{r}\right)$ in equation (4). Equation 3 is transpose rotation matrix direction cosinus matrix $\left(R^{T}\right) \cdot r_{x}$ is $\mathrm{X}$ rows of rotation matrix, $r_{y}$ is $\mathrm{Y}$ rows of rotation matrix and $r_{z}$ is $\mathrm{Z}$ rows of rotation matrix. Rotation matrix $(R)$ obtained from the kinematics of angular motion that occurs when the sensors placed 
on the objects undergo angular displacement of the position. The dot product of $\mathrm{X}$ and $\mathrm{Y}$ rowns of the matrix, which is supposed to be zero and the magnitude of each row and column of $\mathrm{R}$ matrix is approximately equal to one.

2. Correct the $x$ and $y$ column vectors is the DCM as equation (5), $r_{x}^{\prime}$ is $\mathrm{X}$ row of the matrix orthogonal, $r_{y}^{\prime}$ is $\mathrm{Y}$ row of the matrix orthogonal.

3. Normalize these column vectors, using simplified Taylor expansion based method in equation(6). $r_{x}^{\prime \prime}$ is renormalization process to scale $\mathrm{X}$ rows of the $\mathrm{R}$ matrix. $r_{y}^{\prime \prime}$ is renormalization rocess to scale $\mathrm{Y}$ rows of the $\mathrm{R}$ matrix

4. Calculate a new $z$ column vector as equation(7). $r_{z}^{\prime \prime}$ is renormalization rocess to scale $\mathrm{z}$ rows of the $\mathrm{R}$ matrix

5. The reorthogonalized DCM as equation (8). $R^{T \prime}$ is update rotation matrix with reorthogonalized DCM.

Rotation matrix output of DCM $\left(R^{T}\right)$ is converted into a quaternion to eliminate the phenomenon of gimbal lock. This is equation to convert rotation matrix DCM to quarternion[12]:

$$
\begin{aligned}
& q_{3}=0.5 \sqrt{C_{00}+C_{11}+C_{22}+1} \\
& q_{0}=\frac{C_{12}-C_{21}}{4 q_{3}} \\
& q_{1}=\frac{C_{20}-C_{02}}{4 q_{3}} \\
& q_{2}=\frac{C_{01}-C_{10}}{4 q_{3}}
\end{aligned}
$$

With $q_{0}, q_{1}, q_{2}, q_{3}$ are the fundamental quarternion unit. $C_{a b}$ is elements of rotation matrix in row $a$ and column $b$. Gravity of acceleration $\left.\ddot{\left(x_{g}\right.}\right)$ can be calculate from quarternion[13] :

$$
\begin{aligned}
& \ddot{x}_{g x}=2\left(q_{0} q_{2}-q_{3} q_{1}\right) \\
& \ddot{x}_{g y}=2\left(q_{3} q_{0}-q_{1} q_{2}\right) \\
& \ddot{x}_{g z}=q_{3} q_{3}-q_{0} q_{0}-q_{1} q_{1}+q_{2} q_{2}
\end{aligned}
$$

With $\ddot{x}_{g x}$ is gravity of acceleration in $\mathrm{x}$ axis, $\ddot{x}_{g y}$ is gravity of acceleration in Y axis and $\ddot{x}_{g z}$ is gravity of acceleration $Z$ axis. Acceleration of gravity will be used to calculate the linear acceleration as equation(3).

\subsection{Position Estimation Proposed}

Position estimation in general look like in figure 6 . Each stage of the process will have the potential for error processing. So it is very necessary 
correction of each processing to minimize the error occurred. When linear acceleration has been obtained, the position data can be calculated.

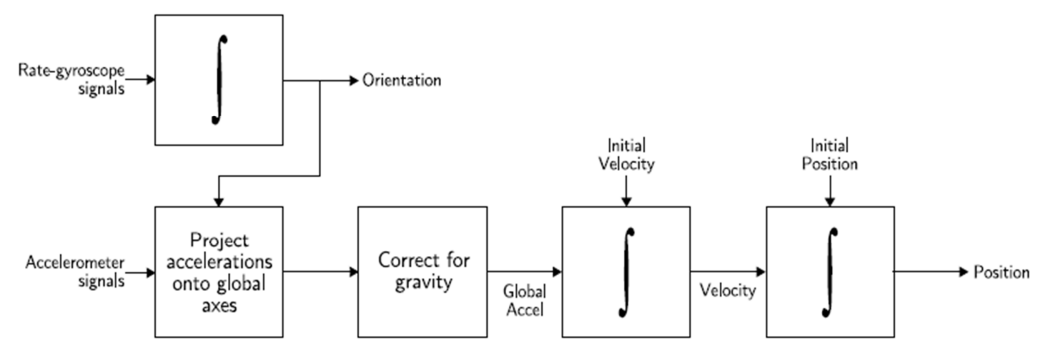

Figure 6. Strapdawn Inertial Navigation System[11]

The challenge in the position estimate is to minimize the error caused by the each process integration in figure 6. Position accuracy degrades with time due to sensor bias drift. An ideal movement detection sensor gives absolute zero velocity periods during which the sensor is stationary state. This is a block diagram of the proposed.

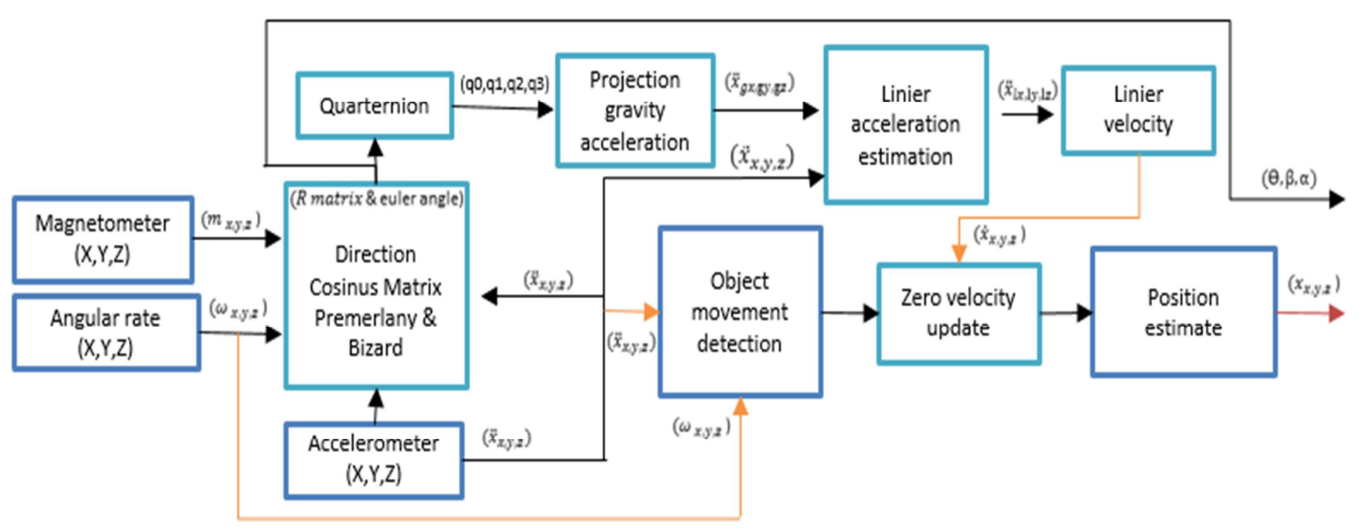

Figure 7. Propose M-ZUPT for position

Output Direction Cosinus Matrix will be convert in quarternion and then calculate projection gravity to get linier acceleration estimation. Object movement detection in our proposed using combine data acceleration with sample variance magnitude acceleration (vm $\ddot{x}$ ) in each axis and angular rate gyroscope. Parameters object movement detection is indicated with orange line in figure 7. Object movement detection have two parameters that is from sensor accelerometer and sensor gyroscope. Sample Variance Magnitude Acceleration(VMA) will be obtained from the processing of accelerometer sensor. Angular Rate(AR) Gyroscope is obtained from a gyro sensor.

$$
m \ddot{x}=\sqrt{\ddot{x}_{x}^{2}+\ddot{x}_{y}^{2}+\ddot{x}_{z}^{2}}
$$




$$
\begin{aligned}
& \overline{m \ddot{x}}=\frac{1}{n} \sum_{i=1}^{n} m \ddot{x}_{i} \\
& v m \ddot{x}^{2}=\frac{1}{n} \sum_{i=1}^{n}\left(m \ddot{x}_{i}-\overline{m \ddot{x}}\right)^{2}
\end{aligned}
$$

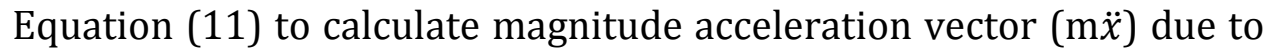
motion for each instant of time. Equation (13) to calculate variance of each magnitude acceleration vector. Mean population of magnitude acceleration vector symbolized by $\overline{m \ddot{x}}$. Zero velocity update(ZUPT) is an effective way to correct low cost inertial measurement Unit (IMU) errors for pedestrian navigation. This is formulation in ZUPT[13]:

Mean velocity $(\overline{\bar{x}})$ can calculate during stationary state.

$$
\overline{\dot{x}}=\frac{\sum_{i=i_{\text {end }}}^{i_{\text {final }}} \dot{x}_{i}}{\left(i_{\text {final }}-i_{\text {end }}\right)}
$$

$t_{\text {end }}$ be the end time of the movement and corresponds to start time fo stationary. $t_{\text {final }}$ be the end time of stationary.

The linier velocity during movement is corrected as below $\left(\dot{x}_{i_{c}}\right)$ :

$$
\dot{x}_{i_{c}}=\dot{x}_{i}-\left.\dot{\dot{x}}\right|_{i=i_{\text {end }} \ldots i_{\text {final }}}
$$

The procedures to obtain each axis $(\mathrm{x}, \mathrm{y}, \mathrm{z})$ linier velocity would be same. The experiment also has been testing modifications ZUPT by variance magnitude acceleration. Modification Zero Velocity Update (ZUPT) looks at the difference detection parameters when the robot moves. The determination of threshold values of VMA conducted trials based on conditions robot on static conditions. On static robots condition (before moving) will experience no change vibration displacement. Data acceleration will appear randomly oscillate at a particular offset value corresponding vibration that occurs in a static condition.

The offset of the measurement results is used as the threshold value movement detection parameter $\left(\tau_{v m \ddot{x}}\right)$. If the value of $\operatorname{VMA}(v \mathrm{vm} \ddot{x})$ is less than the threshold value of VMA $\left(\tau_{v m \cdot x}\right)$, the condition of the object in a stationary state and velocity can be set in zero value. Equation (14)and (15) is equation for Zero Velocity Update (ZUPT).

In the experiment to locate and track legged robot have problem detection of leg robot movement or stationary state. Therefore, object movement detection modifications need to be done to improve performance in estimating velocity ZUPT. In here we use angular velocity to detect of movement leg of robot. Testing is done when the robot walk straight to follow the wall. If velocity angular have positif mean value stationary false, velocity calculation is done. 


\section{EXPERIMENT AND ANALYSIS}

\subsection{Object Movement Detection}

This section is result the performance of the proposed VMA-AR (Varians Magnitude Acceleration) with standart MA (Magnitude Acceleration) and F-MA (Filter-Magnitude Acceleration) for detection object movement. Object using legged robot and the sensor is placed on robotic legs like figure 1.a. Robot move straight along the wall as figure 2. Data Prototype Inertial measurement unit (IMU) will be sent to the computer to do the recording, tracking and analysis. Here is the data submitted by W-IMU.

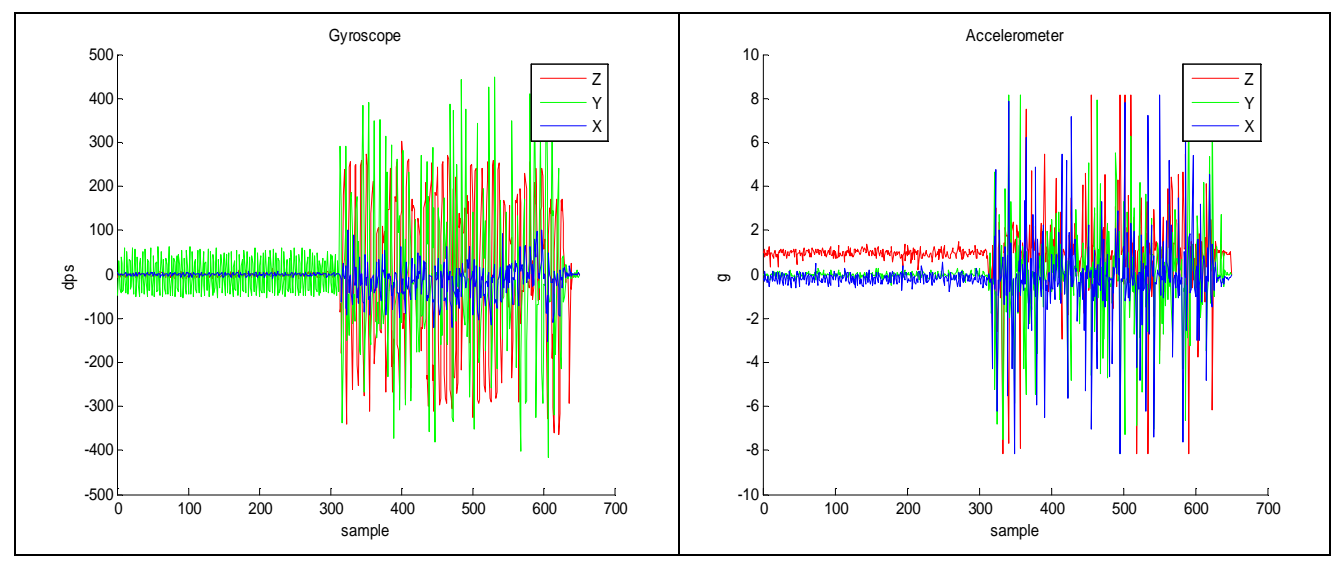

Figure 8. Raw data from the Gyroscope and Accelerometer measured by W-IMU

Robot used in the test, had noise servo on each foot. So that the measurements look like in figure 8. In the static condition, the gyro detects the angular velocity and accelerometer detects acceleration that occurs in all axis.

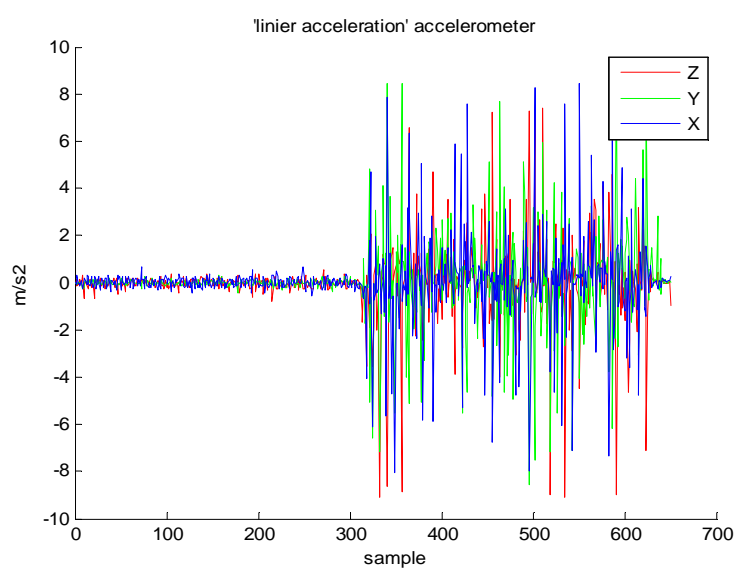

Figure 9. Linier Acceleration Estimation

Linear acceleration is obtained from the equation (3). In figure (9) shows that there is a data error that was sent by W-IMU. W-IMU design just detection acceleration maximum in 8g. Data errors can be caused by the process of wireless data transmission is used. Therefore, the proposed 
method for reducing data errors in the measurement of the object that has a high vibration, it takes a combination of other sensors for detecting robot moving or in a state of static. Based on the experimental motion robot used a combination of accelerometer and gyro sensor to distinguish the robot is moving or static.

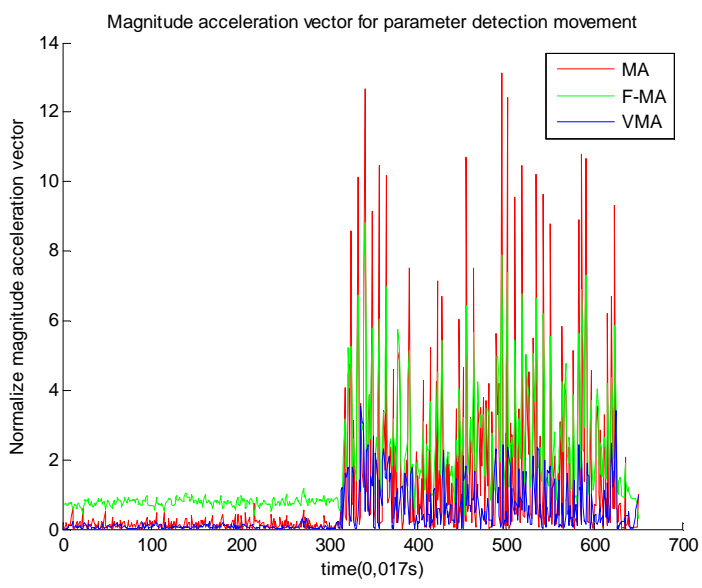

Figure 10. Magnitude Acceleration vector to detect movement.

Conventional movement detection generally use Magnitude Acceleration(MA). Magnitude Acceleration is basically computed from square sum of 3axis acceleration. If calculations MA not equal 1, robots in movement condition. F-MA is a Filtered MA. F-MA is use HPF filter to movement detection. Significant changes in the frequency to be passed and the robot is stated to have movement. VMA is variance magnitude acceleration vector. ). If the value of $\operatorname{VMA}(v \mathrm{~m} \ddot{x})$ is less than the threshold value of VMA $\left(\tau_{v m \ddot{x}}\right)$, the condition of the object in a stationary state and velocity can be set in zero value.

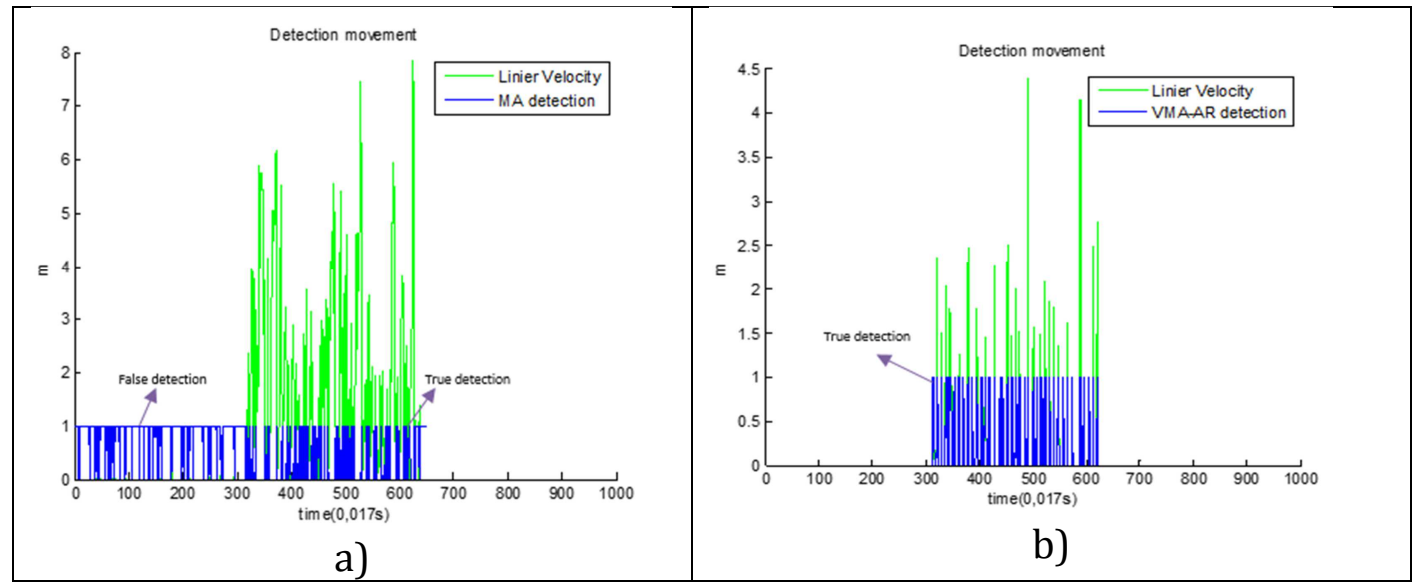

Figure 11. Magnitude Acceleration vector to movement detection in dynamic environtment robot : a) Result movement detection using MA, b) Result movement detection using VMA-AR 
MA detection in static condition there is a detection error. This is due to dynamic environmental conditions on the robot. The true detection in static condition can be seen in Figure 11b.

\subsection{Velocity and Position Estimation}

Linear velocity in Figure 12 is shown by the results of the detection of moving objects using the magnitude of acceleration along with a combination of magnitude acceleration with angular rate gyro.

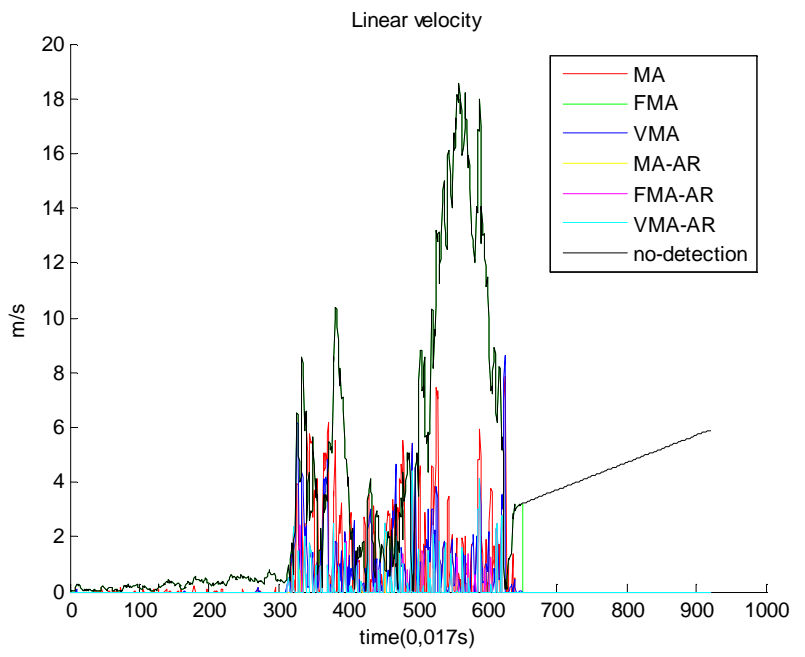

Figure 12. Linier velocity

The results of linear velocity without first movement detection algorithms are accumulative error. Linear position obtained by integrating with respect to linear velocity.

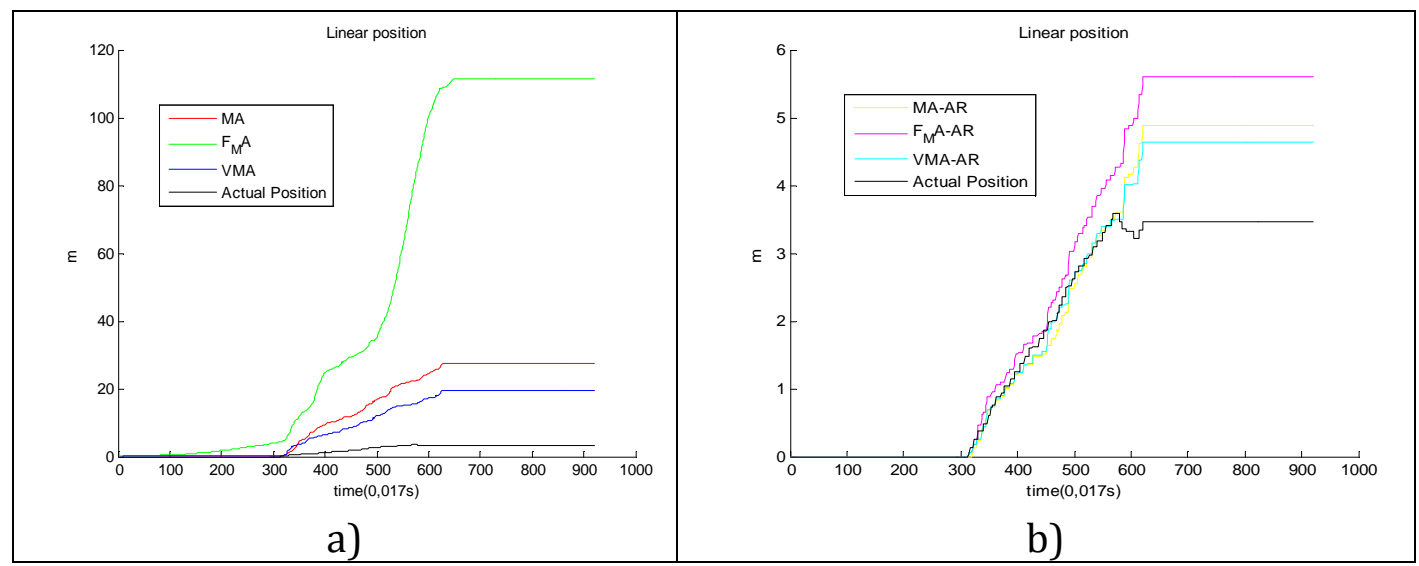

Figure 13. The estimation of linear position a) Linier position with MA,FMA and VMA , b) ) Linier position with MA-AR, FMA-AR, VMA-AR detection.

From the experimental results show that the combination VMA-AR gives the best position estimation. Wireless IMU is placed on robotic legs 
when moving will cause the linear velocity or angular velocity. The most precise method that can be used to detect the movement of the robot is a combination VMA-AR.

Table 1. Statistical analysis of position estimation using Inertial Measurement Unit

\begin{tabular}{|l|l|l|l|l|}
\hline Methods & $\begin{array}{l}\text { Mean error } \\
\text { (meter) }\end{array}$ & $\begin{array}{l}\text { Max error } \\
\text { (meter) }\end{array}$ & $\begin{array}{l}\text { Min error } \\
\text { (meter) }\end{array}$ & $\begin{array}{l}\text { Std error } \\
\text { (meter) }\end{array}$ \\
\hline MA & 11,84832 & 24,16796 & $-0,01924$ & 10,49302 \\
\hline FMA & 48,71444 & 108,0171 & 0 & 46,90119 \\
\hline VMA & 7,881867 & 16,12025 & $-0,06424$ & 6,953906 \\
\hline MA-AR & 0,471411 & 1,426987 & $-0,39042$ & 0,693802 \\
\hline FMA-AR & 0,856625 & 2,148082 & $-0,08585$ & 0,959013 \\
\hline VMA-AR & 0,400013 & 1,182138 & $-0,3236$ & 0,565275 \\
\hline No methods & 56,8945 & 169,5936 & 0 & 58,53044 \\
\hline
\end{tabular}

VMA-AR gives smallest standard deviation and VMA-AR is suitable for minimizing the error in the estimated position of the dynamic environment robot navigation. IMU data transmission performed for 32.5 seconds. VMAAR obtained an average error of 0,400013 meters in 32.5 seconds, so that the error in the method of VMA-AR by $1,23 \mathrm{~cm} / \mathrm{s}$

\section{CONCLUSION}

Minimize error position estimating using inertial measurement unit from double acceleration data has been described. detection of movement on the method of Zero Velocity Update (ZUPT) depending on the patterns of movement. Error position comes from different source such as the prototype IMU hardware, and process estimation linier accelerations and linier position. Error process estimate position can be reduced using detection movement in dynamic environtment. Experiment result shows that prototype IMU with detector VMA-AR obtained error rate $1,23 \mathrm{~cm} / \mathrm{s}$ in the dinamic environtment legged robot.

\section{REFERENCES}

[1] Höflinger, F., Zhang, R., \& Reindl, L. M., Indoor-localization system using a micro-inertial measurement unit (IMU), In European Frequency and Time Forum (EFTF), pp. 443-447, 2012.

[2] Baldwin, G., Mahony, R., Trumpf, J., Hamel, T., \& Cheviron, T., Complementary filter design on the Special Euclidean group SE (3). In Control Conference (ECC), European, pp. 3763-3770, 2007

[3] Madgwick Sebastian., An efficient orientation filter for inertial and inertial/magnetic sensor arrays. Report $x$-io and University of Bristol (UK), 2010

[4] Premerlany William, Bizard Paul, Direction cosine matrix imu: Theory. DIY DRONE: USA, pp. 13-15, 2009 
[5] Wang, Zhelong, et al., Stance-phase detection for ZUPT-aided footmounted pedestrian navigation system, IEEE/ASME Transactions on Mechatronics, pp.3170-3181, 2015

[6] Romaniuk, Sławomir, and Zdzisław Gosiewski, Kalman filter realization for orientation and position estimation on dedicated processor, acta mechanica et automatic, pp. 88-94, 2014

[7] Zhang, Rui, Fabian Hoflinger, and Leonhard Reindl. Inertial sensor based indoor localization and monitoring system for emergency responders, IEEE Sensors Journal, pp. 838-848, 2013

[8] Walchko K. J., Nechyba M. C., Schwartz E., and Arroyo A. Embedded low cost inertial navigation system. In Florida Conference on Recent Advances in Robotics, FAU, Dania Beach, pp. 8-9, 2003

[9] Wang Jeen-Shing, Hsu Yu-Liang, Liu Jiun-Nan, An inertialmeasurement-unit-based pen with a trajectory reconstruction algorithm and its applications, IEEE Transactions on Industrial Electronics, pp. 3508-3521, 2010

[10] Casanova, Oscar Laureano. Robot position tracking using kalman filter, Proceedings of the World Congress on Engineering 2008, London,U.K,Vol II, 2008

[11] Woodman, Oliver J., An introduction to inertial navigation., University of Cambridge, Computer Laboratory, Technical Report No.696, ISSN.1476-2986, 2007

[12] Bak Thomas, Lecture notes-modeling of mechanical systems, Department of Control Engineering , 2002

[13] Abdulrahim, Khairi, et al., Understanding the performance of zero velocity updates in MEMS-based pedestrian navigation., International Journal of Advancements in Technology, pp.53-60, 2014 